\title{
Noether Symmetry Approach for Dirac-Born-Infeld Cosmology
}

\author{
Salvatore Capozziello ${ }^{1,2,3}$, Mariafelicia De Laurentis ${ }^{4 *}$, Ratbay Myrzakulov ${ }^{5}$, \\ ${ }^{1,2}$ Dipartimento di Fisica, Università di Napoli "Federico II", \\ Compl. Univ. di Monte S. Angelo, Edificio G, Via Cinthia, I-80126, Napoli, Italy \\ INFN Sezione di Napoli, Compl. Univ. di Monte S. Angelo, Edificio G, Via Cinthia, I-80126, Napoli, Italy. \\ ${ }^{3}$ Gran Sasso Science Institute (INFN), Via F. Crispi 7, I-67100, L' Aquila, Italy. \\ ${ }^{4}$ Tomsk State Pedagogical University, 634061 Tomsk and National Research Tomsk State University, 634050 Tomsk, Russia and \\ 5 Eurasian International Center for Theoretical Physics and Department of General Theoretical Physics, \\ Eurasian National University, Astana 010008, Kazakhstan.
}

(Dated: July 28, 2021)

\begin{abstract}
We consider the Noether Symmetry Approach for a cosmological model derived from a tachyon scalar field $T$ with a Dirac-Born-Infeld Lagrangian and a potential $V(T)$. Furthermore, we assume a coupled canonical scalar field $\phi$ with an arbitrary interaction potential $B(T, \phi)$. Exact solutions are derived consistent with the accelerated behavior of cosmic fluid.

PACS numbers: 04.50.Kd, 98.80.-k, 95.35. $+\mathrm{d}, 95.36 .+\mathrm{x}$
\end{abstract}

\section{INTRODUCTION}

Scalar fields are introduced in cosmology in order to generate dark energy dynamics and overcome shortcomings coming from the standard $\Lambda \mathrm{CDM}$ model. Wide classes of theories involving scalar fields have been considered in literature by introducing different kinetic terms both canonical and tachyonic and several interesting results come out in view to address the big puzzle of cosmic acceleration. Such scalar fields, in general, are obtained from particle physics and they can give rise to quintessence or phantom behavior in connection to the fact that they can be canonical or tachyonic [1-7]. In particular, there is much interest in the tachyon cosmology since the presence of tachyon fields is well motivated by string theory [8 -10]. There are several work on the interaction between dark energy (tachyon or phantom) and dark matter, where different kinds of interaction terms are phenomenologically introduced [11 16]. Specifically, tachyon fields come from the D-brane action (Dirac-Born-Infeld type Lagrangian) in string theory and they represent the lowest energy state in unstable Dbrane systems [17 -22]. Features as classical dust can be produced from an unstable D-brane through pressureless gas with finite energy density [23, 24]. Some cosmological effects of a tachyon rolling down to its ground state have been widely discussed also using tachyon matter associated with unstable D-branes showing an interesting equation of state as discussed in [25]. Furthermore, tachyonic matter could provide an explanation for inflation at early epochs contributing to some new form of cosmological dark matter at late epochs [26]. Inflation derived from tachyonic fields has been discussed in Ref. [27, 28]. Furthermore, Dirac-Born-Infeld type action containing invariant curvature function, like $f\left(R, R^{2}, \ldots\right)$ gravity functions are considered in Refs. [29 33.

\footnotetext{
*e-mail address: mfdelaurentis@tspu.edu.ru
}

Despite of these interesting results, dynamical equations deriving from this type of Lagrangians are nonlinear and general analytic solutions do not exist. Because of this fact, the Noether Symmetry Approach could be a suitable method to seek for physically motivated solutions 34 37]. The existence of Noether symmetries leads to a specific form of coupling function and the scalar field potential. Furthermore, symmetries lead to the existence of conserved quantities that allows to reduce dynamics thanks to the presence of cyclic variables [38 41$]$. The technique is used to obtain cosmological models in alternative gravity 42 45]. Black holes solutions have also been obtained by Noether symmetries [46, 47].

This paper is organized as follow. In Sec. II we introduce the Dirac-Born-Infeld type Lagrangian and derive the cosmological equations of motion. The Noether Symmetry Approach is described in Sec. [II] and, in Sec. IV. it is applied to the Dirac-Born-Infeld Lagrangian. Some exact solutions are found. Conclusions are drawn in Sec. V

\section{DIRAC-BORN-INFELD TYPE LAGRANGIAN AND EQUATIONS OF MOTION}

Let us consider a system of two interacting scalar fields, a tachyonic field $T$ and a canonical scalar field $\phi$, described by a Dirac-Born-Infeld type Lagrangian which couples the two fields through a potential $B(T, \phi)$. On the other hand, $V(T)$ is the tachyonic potential. The Lagrangian of the system can be written as [48, 49]

$$
\mathcal{L}=-V(T) \sqrt{1-\partial_{\mu} T \partial^{\mu} T}+\frac{1}{2} \partial_{\mu} \phi \partial^{\mu} \phi-B(T, \phi) .
$$

For a spatially flat Friedman-Robertson-Walker universe, we obtain the following point-like Lagrangian

$$
\mathcal{L}=-a^{3} V(T) \sqrt{1-\dot{T}^{2}}+\frac{1}{2} \dot{\phi}^{2} a^{3}-a^{3} B(T, \phi),
$$

where $a$ is the cosmic scale factor of the universe. The Euler-Lagrange equations give dynamics for $\phi$ and $T$, 
that is

$$
\begin{gathered}
\ddot{\phi}+3 H \dot{\phi}+B_{\phi}(T, \phi)=0 \\
\frac{\ddot{T}}{\dot{T}^{2}-1}+3 H \dot{T}+\frac{V_{T}(T)}{V(T)}+B_{T}(T, \phi) \frac{\sqrt{1-\dot{T}^{2}}}{V(T)}=0
\end{gathered}
$$

where $H=\frac{\dot{a}}{a}$ is the Hubble parameter. The subscripts ' $T$ ' and ' $\phi$ ' indicate derivatives of the potentials $B(T, \phi)$ and $V(T)$ with respect to the two fields. Dot indicates derivatives with respect to the cosmic time. The equation for the energy is

$$
E_{\mathcal{L}}=\frac{V(T) \dot{T}^{2}}{\sqrt{1-\dot{T}^{2}}}+\dot{\phi}^{2}=0
$$

\section{NOETHER SYMMETRY APPROACH}

Solutions for the dynamics given by the Lagrangian (2) can be achieved by selecting cyclic variables related to some Noether symmetry [34 37]. In principle, this approach allows to select models compatible with the symmetry so that the conserved quantities could be considered as a sort of Noether charges. Before going into the details of our model, let us introduce the Noether Symmetry Approach.

Let $\mathcal{L}\left(q^{i}, \dot{q}^{i}\right)$ be a canonical, independent of time and non degenerate point-like Lagrangian where

$$
\frac{\partial \mathcal{L}}{\partial \lambda}=0, \quad \quad \operatorname{det} H_{i j} \equiv\left\|\frac{\partial^{2} \mathcal{L}}{\partial \dot{q}^{i} \partial \dot{q}^{j}}\right\| \neq 0,
$$

with $H_{i j}$ the Hessian matrix related to the Lagrangian $\mathcal{L}$ and dot the derivative with respect to an affine parameter $\lambda$, which, in our case, corresponds to the cosmic time $t$. In analytic mechanics, $\mathcal{L}$ is of the form

$$
\mathcal{L}=K(\mathbf{q}, \dot{\mathbf{q}})-V(\mathbf{q})
$$

where $K$ and $V$ are the kinetic energy and potential energy respectively. We stress that $\mathcal{L}$ could assume more complicated expressions than Eq. (77) as in [38 40]. $K$ is a positive definite quadratic form in $\dot{\mathbf{q}}$. The energy function associated with $\mathcal{L}$ is:

$$
E_{\mathcal{L}} \equiv \frac{\partial \mathcal{L}}{\partial \dot{q}^{i}} \dot{q}^{i}-\mathcal{L}
$$

which, when the Lagrangian is in the form (7), reduces to the total energy $K+V$. In any case, $E_{\mathcal{L}}$ is a constant of motion. Since our cosmological problem has a finite number of degrees of freedom, we are going to consider only point transformations. Any invertible transformation of the 'generalized positions' $Q^{i}=Q^{i}(\mathbf{q})$ induces a transformation of the 'gene-ralized velocities' such that:

$$
\dot{Q}^{i}(\mathbf{q})=\frac{\partial Q^{i}}{\partial q^{j}} \dot{q}^{j}
$$

the matrix $\mathcal{J}=\left\|\partial Q^{i} / \partial q^{j}\right\|$ is the Jacobian of the transformation on the positions, and it is assumed to be nonzero. The Jacobian $\tilde{\mathcal{J}}$ of the induced transformation is easily derived and $\mathcal{J} \neq 0 \rightarrow \tilde{\mathcal{J}} \neq 0$. In general, this condition is not satisfied over the whole space but only in the neighborhood of a point. It is a local transformation. A point transformation $Q^{i}=Q^{i}(\mathbf{q})$ can depend on a (or more than one) parameter. As starting point, we can assume that a point transformation depends on a parameter $\epsilon$, so that $Q^{i}=Q^{i}(\mathbf{q}, \epsilon)$, and that it gives rise to a one-parameter Lie group. For infinitesimal values of $\epsilon$, the transformation is then generated by a vector field: for instance, $\partial / \partial x$ is a translation along the $x$ axis while $x(\partial / \partial y)-y(\partial / \partial x)$ is a rotation around the $z$ axis and so on. In general, an infinitesimal point transformation is re-presented by a generic vector field on $Q$

$$
\mathbf{X}=\alpha^{i}(\mathbf{q}) \frac{\partial}{\partial q^{i}}
$$

The induced transformation (9) is then represented by

$$
\mathbf{X}^{c}=\alpha^{i} \frac{\partial}{\partial q^{i}}+\left(\frac{d}{d \lambda} \alpha^{i}(\mathbf{q})\right) \frac{\partial}{\partial \dot{q}^{j}} .
$$

$\mathbf{X}^{c}$ is called the complete lift of $\mathbf{X}$. A function $F(\mathbf{q}, \dot{\mathbf{q}})$ is invariant under the transformation $\mathbf{X}$ if

$$
L_{X} F \equiv \alpha^{i}(\mathbf{q}) \frac{\partial F}{\partial q^{i}}+\left(\frac{d}{d \lambda} \alpha^{i}(\mathbf{q})\right) \frac{\partial}{\partial \dot{q}^{j}} F=0,
$$

where $L_{X} F$ is the Lie derivative of $F$. Specifically, if $L_{X} \mathcal{L}=0, \mathbf{X}$ is a symmetry for the dynamics derived by $\mathcal{L}$. At this point let us consider a Lagrangian $\mathcal{L}$ and its Euler-Lagrange equations

$$
\frac{d}{d \lambda} \frac{\partial \mathcal{L}}{\partial \dot{q}^{j}}-\frac{\partial \mathcal{L}}{\partial q^{j}}=0 .
$$

Let us consider also the vector field (11) and, contracting (13) with $\alpha^{i}$ s, it gives

$$
\alpha^{j}\left(\frac{d}{d \lambda} \frac{\partial \mathcal{L}}{\partial \dot{q}^{j}}-\frac{\partial \mathcal{L}}{\partial q^{j}}\right)=0 .
$$

Since we have

$$
\alpha^{j} \frac{d}{d \lambda} \frac{\partial \mathcal{L}}{\partial \dot{q}^{j}}=\frac{d}{d \lambda}\left(\alpha^{j} \frac{\partial \mathcal{L}}{\partial \dot{q}^{j}}\right)-\left(\frac{d \alpha^{j}}{d \lambda}\right) \frac{\partial \mathcal{L}}{\partial \dot{q}^{j}},
$$

from Eq. (14), it results that

$$
\frac{d}{d \lambda}\left(\alpha^{j} \frac{\partial \mathcal{L}}{\partial \dot{q}^{j}}\right)=L_{X} \mathcal{L} .
$$

The immediate consequence is the Noether theorem which states: if $L_{X} \mathcal{L}=0$, then the function

$$
\Sigma_{0}=\alpha^{k} \frac{\partial \mathcal{L}}{\partial \dot{q}^{k}}
$$


is a constant of motion. Some mathematical comments are necessary now. Eq. (17) can be expressed independently of coordinates as a contraction of $\mathbf{X}$ by a Cartan 1 -form

$$
\theta_{\mathcal{L}} \equiv \frac{\partial \mathcal{L}}{\partial \dot{q}^{j}} d q^{j}
$$

For a generic vector field $\mathbf{Y}=y^{i} \partial / \partial x^{i}$, and 1-form $\beta=\beta_{i} d x^{i}$, we have, by definition, $i_{Y} \beta=y^{i} \beta_{i}$. Thus Eq. (17) can be expressed as:

$$
i_{X} \theta_{\mathcal{L}}=\Sigma_{0}
$$

Through a point transformation, the vector field $\mathbf{X}$ becomes:

$$
\tilde{\mathbf{X}}=\left(i_{X} d Q^{k}\right) \frac{\partial}{\partial Q^{k}}+\left(\frac{d}{d \lambda}\left(i_{X} d Q^{k}\right)\right) \frac{\partial}{\partial \dot{Q}_{k}} .
$$

We see that $\tilde{\mathbf{X}}$ is still the lift of a vector field defined on the space of configurations. If $\mathbf{X}$ is a symmetry and we choose a point transformations such that

$$
i_{X} d Q^{1}=1 ; \quad i_{X} d Q^{i}=0 \quad i \neq 1,
$$

we get

$$
\tilde{\mathbf{X}}=\frac{\partial}{\partial Q^{1}} ; \quad \frac{\partial \mathcal{L}}{\partial Q^{1}}=0 .
$$

Thus $Q^{1}$ is a cyclic coordinate and the dynamics results reduced. Furthermore, the change of coordinates given by (21) is not unique and then a clever choice could be very important. In general, the solution of Eq. (21) is not defined on the whole space. It is local in the sense explained above. Considering the specific case which we are going to analyze, the situation is the following. The Lagrangian is an application

$$
\mathcal{L}: \mathcal{T} \mathcal{Q} \rightarrow \mathbb{R}
$$

where $\mathbb{R}$ is the set of real numbers and the generator of symmetry is

$$
\mathbf{X}=\alpha \frac{\partial}{\partial T}+\beta \frac{\partial}{\partial \phi}+\gamma \frac{\partial}{\partial a}+\dot{\alpha} \frac{\partial}{\partial \dot{T}}+\dot{\beta} \frac{\partial}{\partial \dot{\phi}}+\dot{\gamma} \frac{\partial}{\partial \dot{a}}
$$

As discussed above, a symmetry exists if the equation $L_{X} \mathcal{L}=0$ has solutions. Then there will be a constant of motion on shell, i.e. for the solutions of the Euler equations, as stated above Eq. (17). In other words, a symmetry exists if at least one of the functions $\alpha, \beta$ or $\gamma$ in Eq. 24 is different from zero.

\section{NOETHER SYMMETRY APPROACH FOR DIRAC-BORN-INFELD TYPE-LAGRANGIAN}

Let us apply now the method described in the previous section to the Lagrangian in Eq. (2). From the statement
$L_{X} \mathcal{L}=0$, we obtain:

$$
\begin{aligned}
& \alpha\left[-a^{3} V_{T}(T) \sqrt{1-\dot{T}^{2}}-a^{3} B_{T}(T, \phi)\right]-a^{3} \beta B_{\phi}(T, \phi) \\
& +3 a^{2} \gamma\left[-V(T) \sqrt{1-\dot{T}^{2}}+\frac{1}{2} \dot{\phi}^{2}-B(T, \phi)\right] \\
& +\left(\dot{\phi} \partial_{\phi} \alpha+\dot{T} \partial_{T} \alpha+\dot{a} \partial_{a} \alpha\right)\left(\frac{a^{3} V(T) \dot{T}}{\sqrt{1-\dot{T}^{2}}}\right) \\
& +\left(\dot{\phi} \partial_{\phi} \beta+\dot{T} \partial_{T} \beta+\dot{a} \partial_{a} \beta\right)\left(\dot{\phi} a^{3}\right)=0 .
\end{aligned}
$$

Setting to zero the coefficients of the terms $\dot{\phi}^{2}, \dot{T}^{2}, \dot{\phi} \dot{T}$, $\dot{a} \dot{\phi}$ and $\dot{a} \dot{T}$, we obtain respectively:

$$
\begin{gathered}
\frac{3}{2} \gamma a^{2}+a^{3} \frac{\partial \beta}{\partial \phi}=0 \\
\frac{\partial \alpha}{\partial T}=0 \\
a^{3} \frac{\partial \beta}{\partial T}+\left(\frac{a^{3} V(T) \dot{T}}{\sqrt{1-\dot{T}^{2}}}\right) \frac{\partial \alpha}{\partial \phi}=0
\end{gathered}
$$

$$
\frac{\partial \beta}{\partial a}=0, \quad \frac{\partial \alpha}{\partial a}=0
$$

Finally we have to satisfy the constraint

$$
\begin{aligned}
& a \alpha\left[V_{T}(T) \sqrt{1-\dot{T}^{2}}-B_{T}(T, \phi)\right]+a \beta B_{\phi}(T, \phi)+ \\
+ & 3 \gamma\left[V(T) \sqrt{1-\dot{T}^{2}}+B(T, \phi)\right]=0
\end{aligned}
$$

Eqs. (26)-(29) are consistent for constant values

$$
\begin{aligned}
& \alpha(a, \phi, T)=\alpha_{0}, \\
& \beta(a, \phi, T)=\beta_{0},
\end{aligned}
$$

and finally

$$
\gamma(a, \phi, T)=0
$$

The existence of non-zero quantities $\alpha$ and $\beta$ accounts for the Noether symmetry, provided that they satisfy the constraint (30), which now becomes

$$
\alpha_{0}\left[V_{T}(T) \sqrt{1-\dot{T}^{2}}+B_{T}(T, \phi)\right]+\beta_{0} B_{\phi}(T, \phi)=0 .
$$

It is evident that this constraint makes a direct connection between the tachyon potential $V(T)$ and the potential $B(T, \phi)$. Finally, we find the constant of motion, namely the Noether charge, as

$$
\Sigma_{0}=\alpha_{0}\left(\frac{a^{3} V(T) \dot{T}}{\sqrt{1-\dot{T}^{2}}}\right)+\beta_{0} a^{3} \dot{\phi}
$$


As we can see the constant of motion connects directly the dynamical evolution of the scalar field $\phi$ and the tachyon field $T$ only through the tachyonic potential $V(T)$, and not the coupled potential $B(T, \phi)$. Considering the system of Eqs. (3), (44) and (34), we can find some particular solutions making appropriate positions. In fact supposing that the tachyon scalar field does not depend on time, being $T=T_{0}$ with $T_{0}$ a constant, the system of equations is solved for

$$
\begin{aligned}
\phi(t) & =\phi_{0} e^{n t}, \\
a(t) & =a_{0} e^{-\frac{1}{3} n t}, \\
B_{\phi} & =0, \\
B_{T}\left(T_{0}\right) & =-V_{T}\left(T_{0}\right),
\end{aligned}
$$

where $\phi_{0}$ and $n$ are arbitrary constants while $a_{0}=\left(\frac{\Sigma_{0}}{\beta_{0} \phi_{0} n}\right)^{\frac{1}{3}}$. A second solution can be achieved considering the tachyon scalar field to be constant, being $T=T_{0}$, and $\Sigma_{0}=\beta_{0}=0$. In this case the system of equations is solved for

$$
\begin{aligned}
\phi(t) & =\phi_{0} e^{n t}, \\
a(t) & =a_{1} e^{-\left(\frac{2+n^{2}}{3 n}\right) t}, \\
B_{T} & =0 \\
B(t) & =B_{0} e^{2 n t}, \\
V & =V_{0},
\end{aligned}
$$

where $\phi_{0}, n, a_{1}, B_{0}$ and $V_{0}$ are arbitrary constants. Clearly, the expansion explicitly depends on $n$. In Figs. 1 and 2 are showed the trends of the potential and of the scale factor for different values of $n$. We are adopting a dimensionless time parameter where $t=1$ corresponds to a Hubble time $H_{0}^{-1}$.

\section{CONCLUSIONS}

In this paper, we have obtained the dynamics equations and investigated the conditions for the existence of a Noether symmetry in a Dirac-Born-Infeld type Lagrangian with a tachyonic potential $V(T)$ coupled to a canonical scalar field $\phi$ through an arbitrary interaction potential $B(T, \phi)$. We have shown that a Noether symmetry exists and is related to a constant of motion. Then, we have considered two particular cases where the tachyon scalar field $T$ is a constant and solved the system of equations. In the first case, it is possible to find particular solutions for the system of Eqs. (3), (4) and (34), obtaining the explicit behavior of the scale factor $a(t)$ and of the canonical scalar field $\phi(t)$. As it is clear in the solutions (36), both of them have exponential behavior, while the coupling potential $B$ is independent of $\phi$. From the condition existing between the derivatives of $B$ and $V$ with respect $T$, calculated in $T=T_{0}$, it is possible to obtain a constant value for the tachyonic field. In the
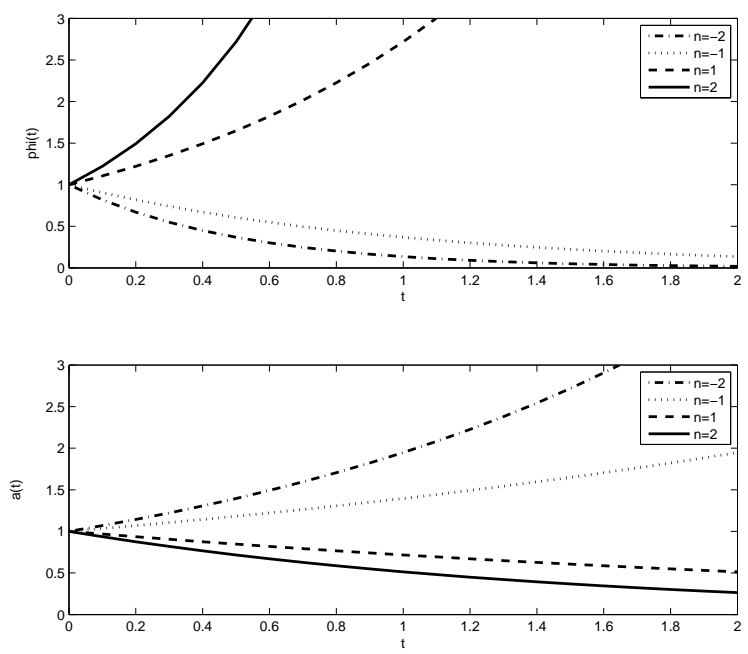

Figure 1: In the top figure is plotted the behavior of $\phi(t)$ and in the bottom figure the behavior of $a(t)$ for different values of $n$ respectively for the solution (36). The solid lines represent the behavior for $n=-2$, the dashed for $n=-1$, the dotter for $n=1$ and dashed-dot for $n=2$.
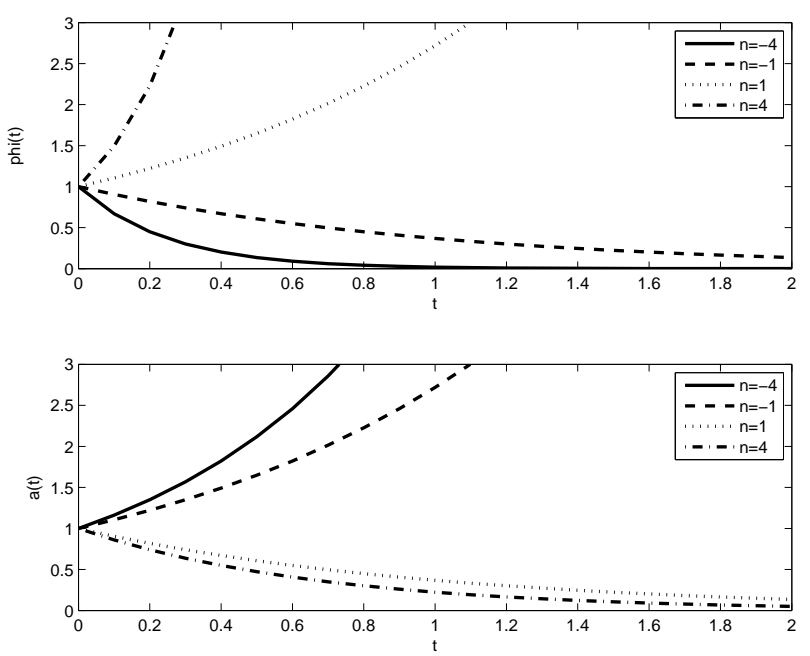

Figure 2: Plots of $\phi(t)$ and $a(t)$ for the solution (37) for different values of $n$. The solid lines represent the behavior for $n=-4$, the dashed for $n=-1$, the dotter for $n=1$ and dashed-dot for $n=4$

second case we find the solutions (37), where $a(t), \phi(t)$ and $B(t)$ are exponential functions. In a forthcoming paper, we will develop these considerations to more general potentials $V(T)$ and $B(T, \phi)$. 
[1] A. de la Macorra and G. Piccinelli, General scalar fields as quintessence, Phys Rev. D 61, (2000) 123503.

[2] E. Copeland, M.R. Garousi, M.Sami and S.Tsujikawa, What is needed of a tachyon if it is to be the dark energy? Phys. Rev.D 71, (2005) 043003.

[3] A.de la Macorra, U.Filobello, G.Germán, The Mass, normalization and late time behavior of the tachyon field, Physics Letters B 635, (2006) 355 .

[4] A.V.Frolov, L. Kofman, A.A Starobinsky, Prospects and problems of tachion matter cosmology, Phys. Lett. B $\mathbf{5 4 5}$, (2002) 8 .

[5] L. Wang, R.R Caldwell, J. P. Ostriker and P.J Steinhardt, Cosmic concordance and quintessence, Astrophys. J. 530, (2000) 17.

[6] I.Zlatev, L.Wang, P.J Steinhardt, Quintessence, cosmic coincidence, and the cosmological constant, Phys.Rev.Lett. 82, (1999) 896.

[7] P.J Steinhardt, L.Wang, I.Zlatev, Cosmological tracking solutions, Phys.Rev.D 59, (1999) 123504.

[8] S. Chattopadhyay, U. Debnath Holographic Dark Energy Model with Modified Variable Chaplygin Gas, Braz.J.Phys. 39 , (2009) 86.

[9] A. Feinstein, Power law inflation from the rolling tachyon, Phys. Rev. D 66, (2002) 063511.

[10] M. Sami, Implementing power law inflation with rolling tachyon on the brane, Mod. Phys. Lett. A 18,(2003) 691

[11] L. Parker and A. Raval, Nonperturbative effects of vacuum energy on the recent expansion of the universe, Phys. Rev. D 60, (1999) 063512.

[12] B. Boisseau, G. Esposito-Farese, D. Polarski and A. A. Starobinsky, Reconstruction of a scalar tensor theory of gravity in an accelerating universe Phys. Rev. Lett. 85, (2000) 2236.

[13] R. R. Caldwell, A Phantom menace? , Phys. Lett. B 545, (2002) 23.

[14] R. Herrera, D. Pavon, W. Zimdahl, Exact solutions for the interacting tachyonic - dark matter system, Gen. Rel. Grav. 36, (2004) 2161.

[15] R. -G. Cai and A. Wang, Cosmology with interaction between phantom dark energy and dark matter and the coincidence problem, JCAP 0503, (2005) 002.

[16] Z.- K. Guo, R.-G. Cai and Y.-Z. Zhang, Cosmological evolution of interacting phantom energy with dark matter, JCAP 0505, (2005) 002 .

[17] M. R. Garousi, Tachyon couplings on nonBPS D-branes and Dirac-Born-Infeld action, Nucl. Phys. B 584, (2000) 284 .

[18] M. R. Garousi, On shell S matrix and tachyonic effective actions, Nucl. Phys. B 647, (2002) 117.

[19] M. R. Garousi, Slowly varying tachyon and tachyon potential, JHEP 0305, (2003) 058 .

[20] Becker K., Becker M. and Schwarz J., String Theory and $M$-Theory (Cambridge: Cambridge University Press) (2007).

[21] B. Zwiebach, A first course in String Theory, (Cambridge: Cambridge University Press) (2009).

[22] M. Green, J. Schwarz, E. Witten, Superstring Theory,(Cambridge: Cambridge University Press) (1987).

[23] A. Sen, Rolling tachyon , JHEP 0204,(2002) 048.

[24] A. Sen, Tachyon matter, JHEP 0207, (2002) 065.
[25] G. W. Gibbons, Cosmological evolution of the rolling tachyon, Phys. Lett. B 537,(2002) 1.

[26] M. Sami, P. Chingangbam and T. Qureshi, Aspects of tachyonic inflation with exponential potential, Phys. Rev. D 66, (2002) 043530.

[27] M. Fairbairn and M.H.G. Tytgat, Inflation from a tachyon fluid?, Phys. Lett. B 546, (2002) 1.

[28] T. Padmanabhan, Accelerated expansion of the universe driven by tachyonic matter Phys. Rev. D 66, (2002) 021301. 528

[29] S. D. Odintsov, G. J. Olmo, D. Rubiera-Garcia, BornInfeld gravity and its functional extensions, Phys. Rev. D 90, (2014), 044003.

[30] M. Jamil, F. M. Mahomed and D. Momeni, Noether Symmetry Approach in $\mathrm{f}(\mathrm{R})$ ?Tachyon Model Phys. Lett. B 702, 315 (2011).

[31] S. Capozziello, M. De Laurentis, Extended Theories of Gravity, Phys. Rept. 509, (2011) 167.

[32] S. Nojiri, S. D. Odintsov, Unified cosmic history in modified gravity: from $\mathrm{F}(\mathrm{R})$ theory to Lorentz non-invariant models Phys. Rept. 505, (2011), 59 .

[33] S. Capozziello, M. De Laurentis, V. Faraoni, A bird's eye view of $f(R)$-gravity The Open Astr. Jour, 2, (2009) 1874.

[34] S. Capozziello, R. De Ritis, C. Rubano, P. Scudellaro, Noether Symmetries in Cosmology, Riv. Nuovo Cim. 19 N4, (1996) 1.

[35] S. Capozziello and A. De Felice, f(R) cosmology by Noether's symmetry, Journal of Cosmology and Astroparticle Physics, 0808, (2008) 016.

[36] S. Capozziello S. and G. Lambiase, Higher order corrections to the effective gravitational action from Noether symmetry approach Gen. Rel. Grav.32, (2000) 295 .

[37] S. Capozziello and G. Lambiase, Double Scalar-Tensor Gravity Cosmologies, Grav.Cosmol. 6, (2000) 164.

[38] S. Capozziello, M. De Laurentis and S. D. Odintsov, Noether Symmetry Approach in Gauss-Bonnet Cosmology, Mod. Phys. Lett. A 29, (2014) 1450164.

[39] S. Basilakos, S. Capozziello,M. De Laurentis, A. Paliathanasis, M. Tsamparlis, Noether symmetries and analytical solutions in $\mathrm{f}(\mathrm{T})$-cosmology: A complete study, Phys. Rev. D 88, (2013) 103526.

[40] S. Capozziello, M. De Laurentis, Noether symmetries in extended gravity quantum cosmology Int. J. Geom. Meth. Mod. Phys. 11, (2014) 1460004.

[41] S. Capozziello, M. De Laurentis, R. Myrzakulov, Noether Symmetry Approach for teleparallel-curvature cosmology, arXiv:1412.1471 [gr-qc] (2014).

[42] M. Sharif, S. Waheed, Noether symmetries of some homogeneous universe models in curvature corrected scalartensor gravity, JCAP 02 (2013) 043.

[43] U. Camci, Y. Kucukakca, Noether symmetries of Bianchi I, Bianchi III, and Kantowski-Sachs spacetimes in scalarcoupled gravity theories, Phys. Rev. D 76, (2007) 084023.

[44] M. Sharif, S. Waheed, Re-scaling of energy in stringy charged black hole solutions using approximate symmetries, Can. J. Phys. 88, (2010) 833.

[45] M. Sharif, S. Waheed, Energy of Bardeen Model Using Approximate Symmetry Method Phys. Scripta 83, (2011) 015014 .

[46] S. Capozziello S., Stabile A. and Troisi A., Spherically 
symmetric solutions in $\mathrm{f}(\mathrm{R})$-gravity via Noether Symmetry Approach, Class. Quantum Grav.24, (2007) 2153.

[47] S. Capozziello, M. De Laurentis, A. Stabile,Axially symmetric solutions in $\mathrm{f}(\mathrm{R})$-gravity, Class.Quant.Grav. 27, (2010) 165008.

[48] A de la Macorra and U. Filobello, Interacting Tachyon:
Generic cosmological evolution for a tachyon and a scalar field, Phys.Rev.D 77, (2008) 023531.

[49] U. Filobello, A. de la Macorra, Accelerating Cosmological Models for an Interacting Tachyon, Nuovo Cim.B 125, (2010) 315 . 